YURI, J.E.; RESENDE, G.M.; MOTA, J.H.; RODRIGUES JÚNIOR, J.C.; SOUZA, R.J.; CARVALHO, J.G. Comportamento da alface americana em função do uso de doses e épocas de aplicação de boro em cultivo de inverno. Horticultura Brasileira, Brasília, v.22, n.3, p.593-596, ,jul-set 2004.

\title{
Comportamento da alface americana em função do uso de doses e épo- cas de aplicação de boro em cultivo de inverno
}

\author{
Jony E. Yuri' ${ }^{1}$; Geraldo M. de Resende ${ }^{2}$; José H. Mota ${ }^{1}$; Juarez C. Rodrigues Júnior ${ }^{3}$; Rovilson J. de \\ Souza $^{1}$; Janice G. de Carvalho ${ }^{4}$ \\ ${ }^{1}$ UFLA, Depto. Agricultura, C. Postal 37, Lavras-MG; ${ }^{2}$ Embrapa Semi-Árido, C. Postal 23, 56300-970 Petrolina-PE; ${ }^{3}$ REFRICON, Rod. \\ Regis Bittencourt s/n km 294, 06850-000 Itapecerica da Serra-SP. ${ }^{4}$ UFLA, Depto. Ciências do Solo; E-mail: jonyyuri@uol.com.br
}

\section{RESUMO}

Avaliou-se a influência de doses de bórax sobre o rendimento e qualidade pós-colheita da alface americana (Lactuca sativa L.), em três ensaios distintos conduzidos de junho a agosto/2002, no município de Três Pontas (MG). Utilizou-se o delineamento de blocos ao acaso com cinco doses de bórax $(0,00 ; 0,75 ; 1,50 ; 2,25 \mathrm{e}$ $\left.3,00 \mathrm{~kg} \mathrm{ha}^{-1}\right)$ e quatro repetições, aplicadas em três diferentes épocas via foliar (14; 21 e 28 dias após o transplantio). A massa fresca total apresentou efeito quadrático no qual a dose de $1,58 \mathrm{~kg} \mathrm{ha}^{-1} \mathrm{de}$ bórax resultou em maior rendimento. Verificou-se variação de 795 a $829 \mathrm{~g} \mathrm{planta}^{-1}$ entre as épocas de aplicação, sem diferirem estatisticamente entre si. A aplicação de $1,71 \mathrm{~kg} \mathrm{ha}^{-1}$ de bórax possibilitou a obtenção de maior massa fresca comercial. O melhor resultado (563 g planta $\left.^{-1}\right)$ foi obtido na aplicação aos 21 dias. Esta data também contribuiu na obtenção de maior circunferência da cabeça $(41,7 \mathrm{~cm})$, sendo significativamente superior à aplicação aos 28 dias (40,6 cm). Não foram observados efeitos significativos para o comprimento de caule entre os tratamentos. A dose de $1,55 \mathrm{~kg} \mathrm{ha}^{-1}$ de bórax proporcionou uma melhor conservação da alface mantida durante 20 dias em câmara-fria.

Palavras-chave: Lactuca sativa L., rendimento, bórax, método de aplicação, adubação, conservação pós-colheita.

\begin{abstract}
Behavior of crisp head lettuce as a result of doses and application times of boron in winter crop

The influence of doses of borax on yield and post harvest quality of crisp head lettuce (Lactuca sativa L.) was evaluated. Three distinct trials were conducted in Tres Pontas, Minas Gerais State, Brazil, from June to August 2002. The randomized block design with five doses of borax $\left(0.00 ; 0.75 ; 1.50 ; 2.25\right.$ and $\left.3.00 \mathrm{~kg}^{-h^{-1}}\right)$ and four replicates, applied foliarly at different times $(14 ; 21$ and 28 days after transplanting) was evaluated. The total fresh matter presented a quadratic effect, where the dose of $1.58 \mathrm{~kg} \mathrm{ha}^{-1}$ of borax resulted in a greater yield. A range of 795 to $829 \mathrm{~g} \mathrm{plant}^{-1}$ between different application times, with no statistical significant differences was observed. The application of $1.71 \mathrm{~kg} \mathrm{ha}^{-1}$ of borax resulted in greater commercial fresh matter production. The best results $\left(563 \mathrm{~g} \mathrm{plant}^{-1}\right)$ were obtained when the application was performed at 21 days. Applications at 21 days also resulted in greater head circumference $(41.7 \mathrm{~cm})$, being statistically superior to the applications at 28 days $(40.6 \mathrm{~cm})$. Stem length did not present any significant effects between treatments. The dose of $1.55 \mathrm{~kg} \mathrm{ha}^{-1}$ of borax presented best lettuce conservation in cold-chamber (20 days).
\end{abstract}

Keywords: Lactuca sativa L., yield, borax, application methods, fertilization, post harvest conservation.

\section{(Recebido para publicação em 16 de abril de 2003 e aceito em 20 de fevereiro de 2004)}

A alface americana vem adquirindo importância crescente no país. Seu plantio visa atender as redes fast food e, atualmente, tem-se constatado o aumento no consumo desta hortaliça também na forma de salada. No ano de 2001, entre os diferentes tipos de alface comercializados na CEAGESP, $29,6 \%$ foram representadas pela alface americana (Conjuntural 2001).

Os solos de regiões tropicais chuvosas, apresentam normalmente baixos teores de boro total e disponível, independente do material de origem, o que é ocasionado pela alta mobilidade do elemento no solo e pelo alto grau de intemperismo (Fassbender e Bornemisza, 1994).

Entre os micronutrientes que mais afetam o desenvolvimento da alface, quando em níveis deficientes, são o cobre, molibdênio e boro, em ordem decrescente de intensidade de efeitos sobre o peso médio das cabeças comerciais, promovendo reduções de $95 \%$, $68 \%$ e $23 \%$, respectivamente (Adams et al., 1986). Fontes et al. (1982) também observaram aumento na produção total e no peso médio da cabeça comercial em 7,4 e 6,3\%, respectivamente, quando cultivada em solo com nível satisfatório de boro.

A adubação foliar com ácido bórico promove acréscimos de $17,9 \%$ na produção e $12,1 \%$ na altura de plantas, comparativamente à testemunha, e normalmente apresenta mesma eficiência de tratamento em que o boro é aplicado diretamente no solo, no entanto, sem apresentar diferenças significativas
(Gimenes et al., 1990). Os efeitos positivos da aplicação de boro em outras oleráceas, como na qualidade e produção da batata, são relatados por Mondy e Munshi (1993) e Pregno e Armour (1992). Efeito semelhante foi observado na produção de matéria seca em rabanete (Azevedo, 1999).

Ao contrário, François (1988) verificou que o peso médio total e comercial da cabeça de alface decresceu significativamente com o aumento da concentração de boro. Com $15 \mathrm{mg} \mathrm{L}^{-1}$ de boro, esses pesos foram menores em torno de $25 \%$. A produção da alface foi menor em $1,7 \%$ a cada aumento unitário $\left(\mathrm{mg} \mathrm{L}^{-1}\right)$ de boro na solução acima de $1,3 \mathrm{mg} \mathrm{L}^{-1}$. Estes resultados indicam ser a alface mais sensível que brócolis e rabanete, que se encontram também 


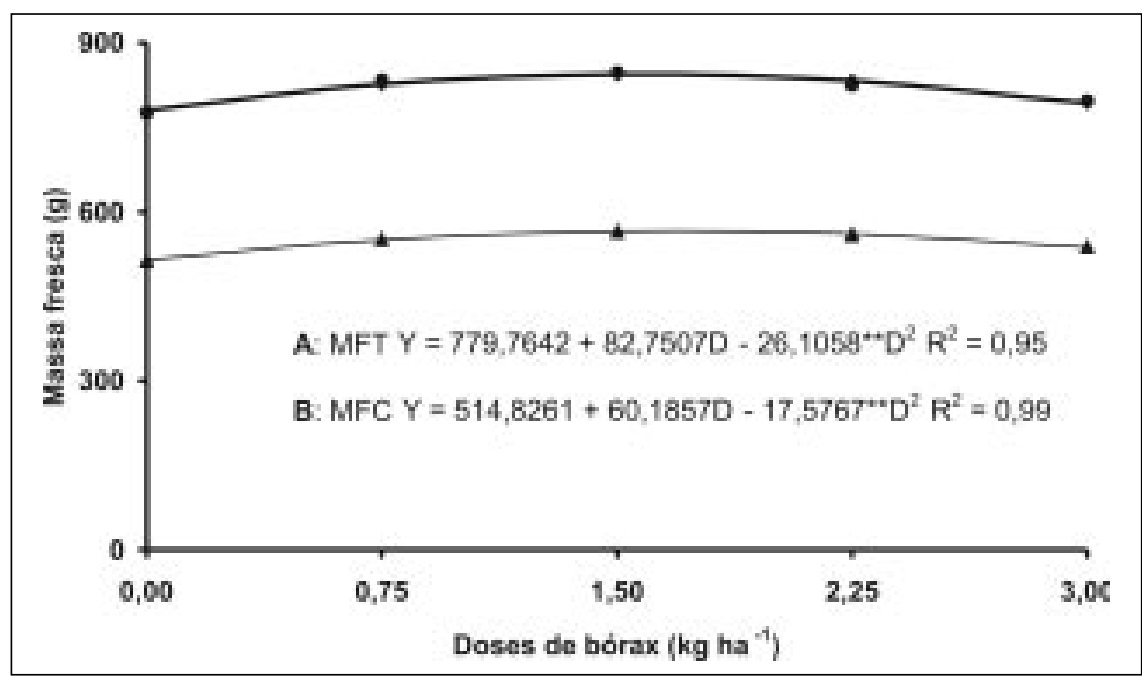

Figura 1. Massa fresca total (MFT) e comercial (MFC) dos três experimentos da alface em função do uso de doses de bórax. Três Pontas (MG), UFLA, 2002.

classificados com moderadamente sensíveis ao boro (François, 1986). De acordo com Havlin et al. (1999), a alface é considerada como de média sensibilidade, quando em condições de baixa disponibilidade no solo.

Os principais sintomas de deficiência de boro em alface são caracterizados por aspereza, enrugamento e coloração prateada das folhas (Weir e Cresswell, 1993).

A falta de informações sobre esse micronutriente na cultura justificou $o$ presente trabalho, que objetivou avaliar o efeito da aplicação foliar de diferentes doses e épocas de aplicação de boro sobre as características produtivas e qualidade pós-colheita da alface americana, produzida em condições de campo.

\section{MATERIAL E MÉTODOS}

Foram conduzidos três ensaios distintos, de junho a agosto 2002, no município de Três Pontas (MG), para avaliar a influência de doses e épocas de aplicação de boro sobre a produção de alface americana (Lactuca sativa $\mathrm{L}$.) $\mathrm{O}$ tipo de solo predominante na área é o LATOSSOLO VERMELHO Distroférrico de textura argilosa. A análise química do solo da área experimental apresentou as características: $\mathrm{K}=100$ $\mathrm{mg} \mathrm{dm}{ }^{-3} ; \mathrm{P}=44,7 \mathrm{mg} \mathrm{dm}^{-3} ; \mathrm{Ca}=4,1$ $\mathrm{cmol}_{\mathrm{c}} \mathrm{dm}^{-3} ; \mathrm{Mg}=0,7 \mathrm{cmol}_{\mathrm{c}} \mathrm{dm}^{-3} ; \mathrm{Al}=$ $0,0 \mathrm{cmol} \mathrm{dm}^{-3} ; \mathrm{H}+\mathrm{Al}=2,1 \mathrm{cmol} \mathrm{dm}^{-3}$; $\mathrm{Zn}=3,8^{\mathrm{c}} \mathrm{mg} \mathrm{dm}^{-3} ; \mathrm{Fe}=29,8 \mathrm{mg} \mathrm{dm}^{-3}$;

, ção, e quatro repetições, aplicadas em três épocas via foliar $(14 ; 21$ e 28 dias após o transplantio), sendo cada época de aplicação considerada um ensaio. As aplicações foliares foram realizadas com pulverizador manual com 4 L de capacidade em máxima pressão, gastandose $300 \mathrm{~L}$ de calda por hectare. Para evitar a ocorrência de deriva, no momento da aplicação, foi instalada ao redor da parcela uma cortina plástica com 1 metro de altura.

As parcelas experimentais constituíram-se de canteiros com quatro linhas de $2,1 \mathrm{~m}$ de comprimento espaçadas de $0,35 \mathrm{~m}$, sendo $0,30 \mathrm{~m}$ a distância entre plantas na linha. As linhas centrais formaram a área útil, onde foram colhidas seis plantas, para a realização das avaliações. Para a adubação de plantio utilizou-se $1700 \mathrm{~kg} \mathrm{ha}^{-1}$ da formula 4-14-8 e $1000 \mathrm{~kg} \mathrm{ha}^{-1}$ de superfosfato simples. As adubações de cobertura foram realizadas por meio de fertirrigações diárias, totalizando $40 \mathrm{~kg} \mathrm{ha}^{-1}$ de $\mathrm{N}$ e $85 \mathrm{~kg} \mathrm{ha}^{-1}$ de $\mathrm{K}$, utilizando a uréia e o cloreto de potássio respectivamente, como fontes de $\mathrm{N}$ e de K (Yuri et al., 2002a). O transplantio das mudas, com 29 dias de do-se diariamente, sendo a cultura conduzida sob mulching. Os demais tratos culturais foram os comuns à cultura.

As colheitas foram feitas em 20/08/ 2002 sendo avaliadas a massa fresca total e comercial (g planta ${ }^{-1}$ ); circunferência e comprimento do caule da cabeça comercial $(\mathrm{cm})$ e conservação póscolheita aos 10 e 20 dias em câmara frigorífica a $5 \pm 2^{\circ} \mathrm{C}$, avaliada através de notas (nota 1: cabeças comerciais extremamente deterioradas; nota 2: cabeças comerciais deterioradas; nota 3: cabeças comerciais moderadamente deterioradas; nota 4: cabeças comerciais levemente deterioradas e nota 5: cabeças comerciais sem deterioração), sendo utilizados três avaliadores e retirada a média das notas obtidas. Os dados foram submetidos à análise de variância conjunta dos experimentos (épocas de aplicação), sendo as médias comparadas pelo teste de Tukey para épocas de aplicação e regressão polinomial para doses, com base no modelo quadrático, ao nível de $5 \%$ de probabilidade.

\section{RESULTADOS E DISCUSSÃO} idade, foi feito em 17/06/2002, irrigan-
Os dados de massa fresca total dos três experimentos quando submetidos à análise de regressão, mostraram que a equação polinomial de segundo grau foi a que apresentou o melhor ajuste $\left(\mathrm{R}^{2}=\right.$ 0,95). A equação de regressão estimada foi $\mathrm{Y}=779,7642+82,7507 \mathrm{D}-$ $26,1058 \mathrm{D}^{2}$, onde $\mathrm{Y}$ representou a massa fresca total ( g planta $^{-1}$ ) e D as doses de boro empregadas. Por meio desta equação o maior rendimento estimado de matéria fresca total foi obtido com o uso de 1,58 $\mathrm{kg} \mathrm{ha}^{-1}$ de bórax (Figura 1), o que representa comparativamente à testemunha, sem aplicação, um incremento em termos de rendimento de $8,2 \%$ por planta. Considerando-se que não há custos da aplicação de bórax, uma vez que o mesmo pode ser realizado em conjunto com os tratos fitossanitários da cultura, o custo total da aplicação seria apenas do preço do micronutriente ( $\mathrm{R} \$ 5,00 / \mathrm{ha}$ ), o que justifica plenamente o seu uso em razão do baixo custo e da relação custo/beneficio. A produção de massa fresca total apresentou variação de 795 a 829 g plan$\mathrm{ta}^{-1}$ entre as diferentes épocas de aplica- 
ção, sem diferirem estatisticamente entre si (Tabela 1). Resultados similares foram encontrados para massa fresca comercial. Quando submetidos à análise de regressão, mostraram que a equação polinomial de segundo grau foi a que apresentou o melhor ajuste $\left(\mathrm{r}^{2}=\right.$ $0,99)$. A equação de regressão estimada foi $\mathrm{Y}=514,8261+60,1857 \mathrm{D}-$ $17,5767 \mathrm{D}^{2}$. Por meio desta equação o maior rendimento estimado de matéria fresca comercial foi obtido com o uso de $1,71 \mathrm{~kg} \mathrm{ha}^{-1}$ de bórax (Figura 1). $\mathrm{O}$ resultado foi significativamente superior quando as aplicações foram efetuadas aos 21 dias, em comparação com 28 dias, com $563 \mathrm{~g} \mathrm{planta}^{-1}$ (Tabela 1). Efeitos positivos da aplicação de boro em alface são relatados por Fontes et al. (1982) e Adams et al. (1986). Também, Gimenes et al. (1990), mencionam que a adubação foliar com ácido bórico promoveu um acréscimo de $17,9 \%$ na produção e $12,1 \%$ na altura de plantas, comparativamente à testemunha, índices superiores aos obtidos nos tratamentos em que se aplicou boro no solo, no entanto, sem diferenças significativas. Ao contrário, François (1988) verificou que os pesos médios total e comercial das cabeças de alface foram significativamente menores com o aumento da concentração de boro. A concentração de 15 $\mathrm{mg} \mathrm{L}^{-1}$ de boro reduziu os pesos médios em torno de $25 \%$, nos anos em estudo.

Observou-se efeito sobre a circunferência da cabeça comercial (Tabela 1) apenas para época de aplicação, onde a aplicação aos 21 dias contribuiu para o maior retorno $(41,7 \mathrm{~cm})$, sendo significativamente superior à aplicação aos 28 dias $(40,6 \mathrm{~cm})$.

O comprimento de caule não proporcionou efeitos significativos entre tratamentos. O comprimento de caule é característica importante na cultura da alface americana, principalmente, quando destinada à indústria de beneficiamento. Deve ser bastante reduzido, para evitar perdas durante o processamento. Segundo Yuri et al. (2002b), caule excessivamente comprido não possui boa compacidade e dificulta o beneficiamento, afetando a qualidade final do produto.

Não se observou diferença significativa para conservação pós-colheita,
Tabela 1. Massa fresca total e comercial $\left(\mathrm{g} \mathrm{planta}^{-1}\right)$, circunferência da cabeça e comprimento de caule $(\mathrm{cm})$ de alface em função da época de aplicação de bórax. Três Pontas (MG), UFLA, 2002.

\begin{tabular}{lccc}
\hline \multirow{2}{*}{ Características } & \multicolumn{3}{c}{ Épocas de aplicação (dias após transplantio) } \\
\cline { 2 - 4 } & $\mathbf{1 4}$ & $\mathbf{2 1}$ & $\mathbf{2 8}$ \\
\hline Massa fresca total & $822 \mathrm{a}$ & $829 \mathrm{a}$ & $795 \mathrm{a}$ \\
Massa fresca comercial & $542 \mathrm{ab}$ & $563 \mathrm{a}$ & $531 \mathrm{~b}$ \\
Circunferência da cabeça & $41,0 \mathrm{ab}$ & $41,7 \mathrm{a}$ & $40,6 \mathrm{~b}$ \\
Comprimento de caule & $5,6 \mathrm{a}$ & $5,7 \mathrm{a}$ & $6,0 \mathrm{a}$ \\
\hline
\end{tabular}

${ }^{1}$ Médias seguidas pela mesma letra nas linhas, não diferem entre si, pelo teste de Tukey ao nível de $5 \%$ de probabilidade.

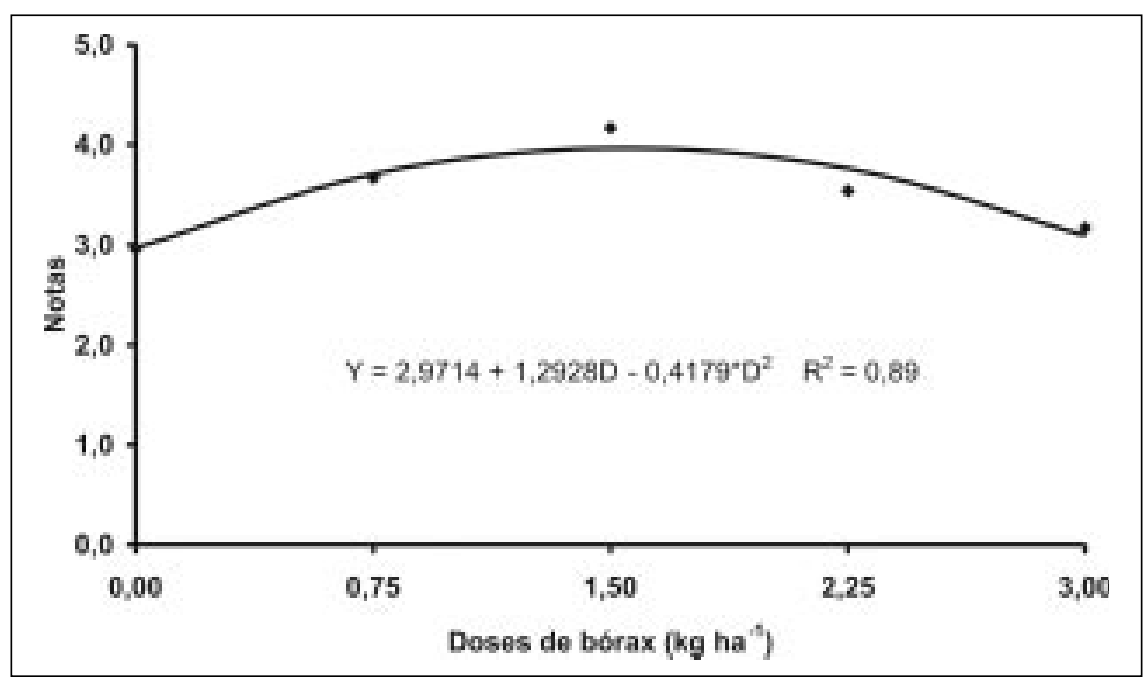

Figura 2. Conservação da cabeça comercial de alface durante 20 dias em câmara-fria, em função do uso de doses de bórax. Três Pontas (MG), UFLA, 2002.

aos 10 dias. Para a conservação aos 20 dias após a colheita a equação estimada foi $\mathrm{Y}=2,9714+1,2928 \mathrm{D}-0,4179 * \mathrm{D}^{2}$ $\left(r^{2}=0,89\right)$. Pela equação foi possível verificar efeito quadrático onde a dose de $1,55 \mathrm{~kg} \mathrm{ha}^{-1}$ de bórax proporcionou a melhor conservação pós-colheita da alface. A literatura consultada não registra informações de conservação pós-colheita em função da aplicação de boro na cultura. O que foi observado na prática é que visualmente o boro parece inibir a oxidação das folhas externas da cabeça de alface comparativamente à testemunha sem aplicação, que se mostrou com uma coloração marrom.

Nas condições em que foi realizado o experimento, conclui-se que a pulverização de $1,71 \mathrm{~kg} \mathrm{ha}^{-1}$ de bórax aos 21 dias após o transplantio proporciona melhor rendimento e qualidade comercial da alface americana, enquanto que para a melhor conservação pós-colheita, a dose mais indicada é de $1,55 \mathrm{~kg} \mathrm{ha}^{-1}$.

\section{LITERATURA CITADA}

ADAMS, P.; GRAVES, C.J.; WINSOR, G.W. Some effects of micronutrients and liming on the yield, quality and micronutrient status of lettuce grown in beds of peat. Journal Horticultural Science, v.61, n.4, p.515-521, 1986.

AZEVEDO, W.R. Níveis críticos de boro em rabanete cultivado em solos de várzea do Sul de Minas Gerais. Lavras: UFLA, 1999. 53 p. (Tese mestrado).

CONJUNTURAL de produtos por agência. CEAGESP. Boletim Mensal. São Paulo. jan./dez. 2001.

FASSBENDER, H.W., BORNEMISZA, E. Quimica de suelos: con énfasis en suelos de América Latina. 2 ed. 1994. 420 p. (Colección de Livros y Materiales Educativos/IICA, n. 81).

FONTES, R.R., LIMA, J.A.; TORRES, A.C.; CARRIJO, O.A. Efeito da aplicação de $\mathrm{Mg}, \mathrm{B}$, Zn e Mo na produção de alface. Pesquisa Agropecuária Brasileira, Brasília, v.17, n.2, p.171175, 1982.

FRANCOIS, L.E. Effect of excess boron on broccoli, cauliflower, and radish. Journal American Society Horticultural Science, v.111, n.4, p.494-498, 1986. 
FRANCOIS, L.E. Yield and quality of celery and crisphead lettuce to excess boron. Journal American Society Horticultural Science, v.113, n.4, p.538-542, 1988.

GIMENES, N.L.; TEIXEIRA, R.R.; CARVA-

LHO, S.L.; TEIXEIRA, N.T. Adubação com boro na cultura da alface (Lactuca sativa L.) cv. Aurélia Repolhuda. Ecossistema, Espírito Santo do Pinhal, v.15, p.145-148, 1990.

HAVLIN, J.L; BEATON, J.D.; TISDALE, S.L.; NELSON, W.L. Soil fertility and fertilizers: an introduction to nutrient management. New Jersey: 6 ed., Prentice Hall, 1999. 499 p.
MONDY, N.I.; MUNSHI, C.B. Effect of boron on enzymatic discolaration and phenolic and ascorbic acid contend of potatoes. Journal Agricultural Food Chemistry, v.41, n.4, p.554-556, 1993.

PREGNO, L.M.; ARMOUR, J.D. Boron deficiency and toxicity in potato cv. Sebago on an oxisol of the Atherton Tablelands, North Queensland. Australian Journal of Experimental Agriculture, v.32, n.2, p.251 -253, 1992.

WEIR, R.G.; CRESSWELL, G.C. Plant nutrient disorders 3. Vegetable crops. Sydney, 1993. 105 p.
YURI J.E.; MOTA, J.H.; SOUZA, R.J.; RESENDE, G.M.; FREITAS, S.A.C.; RODRIGUES JUNIOR, J.C. Alface americana: cultivo comercial. Lavras: UFLA, 2002. 51 p. Texto acadêmico.

YURI, J.E.; SOUZA, R.J.; FREITAS, S.A.C.; RODRIGUES JÚNIOR, J.C.; MOTA, J.H. Comportamento de cultivares de alface tipo americana em Boa Esperança. Horticultura Brasileira, Brasília, v.20, n.2, p.229-232, 2002. 